In Crescendo. Institucional. 2015; 6(1): 64-73

\title{
CARACTERIZACIÓN DEL CONTROL INTERNO \\ EN LA GESTIÓN DE LAS EMPRESAS COMERCIALES DEL PERÚ 2013
}

\section{Characterization of INTERNAL CONTROL IN THE MANAGEMENT OF PERU TRADING ENTERPRISES 2013}

\author{
Daniel Obispo Chumpitaz* \\ Yuri Gagarin Gonzales Rentería*
}

\begin{abstract}
RESUMEN
T a investigación tiene por objetivo identificar y describir la caracterización del control Linterno en la gestión de las empresas comerciales del Perú en el periodo 2013. La investigación fue descriptiva, y para recoger información se utilizaron las fichas bibliográficas. El principal resultado es: el control interno permite evaluar el grado de eficiencia, eficacia, economía y productividad en las empresas del rubro comercial, con lo que se logra en muchos casos alcanzar en un 100 \% sus objetivos y metas programados. También permite minimizar riesgos y errores o irregularidades en un $80 \%$ de forma oportuna, y contar con un adecuado y eficiente control para tomar decisiones en la vida empresarial. La principal conclusión es: la existencia de un control influye en las diferentes áreas de la empresa maximizando oportunidad, eficiencia de las operaciones, rentabilidad, y confiabilidad de la información administrativa, contable y financiera. El entorno o el ambiente forma al personal para que desarrolle sus actividades y cumpla con sus responsabilidades. Las actividades de control se establecen para ayudar a asegurar que se pongan en práctica las reglas para enfrentar cualquier riesgo. Todo este proceso es supervisado para proporcionar un grado de seguridad razonable en los objetivos de la empresa.
\end{abstract}

Palabras claves: administrativos, auditoría, control interno, gestión, servicios.

* Bachiller en Contabilidad, asesor de empresas.

** Doctor en Contabilidad y Finanzas, asesor y consultor de empresas. 


\begin{abstract}
The research aimed to identify and describe the characterization of internal control in the management of business enterprises of Peru in the 2013 period was descriptive research for information gathering bibliographic records was used. The main result is: The internal control to assess the efficiency, effectiveness, economy and productivity in companies achieving commercial grade item in many cases reaching $100 \%$ in its objectives and targets scheduled. It also helps to minimize risks and errors or irregularities in a timely manner $80 \%$ having an adequate and efficient control for decision making in business life. The main conclusion is: The existence of control influences the different areas of the company to maximize opportunity, efficiency of operations, profitability and reliability of administrative, accounting and financial information. The setting and ambience trains staff to develop their activities and fulfill its responsibilities. Control activities are established to help ensure that the rules put in place to address any risk. This whole process is monitored to provide a reasonable degree of certainty as to the objectives of the company.
\end{abstract}

Keywords: administrative, audit, internal control, management, services. 


\section{INTRODUCCIÓN}

Según Bilick (2003), un buen sistema de control interno permite a cualquier organización tener mayores posibilidades de lograr los objetivos que ella fija. Existían muchas y diversas definiciones y opiniones sobre el control interno. Así que se desarrollaron algunas estructuras de control que alcanzaron amplia difusión internacional, principalmente por la obligatoriedad que existe en algunos países: que las organizaciones informen a terceros sobre la eficacia de su sistema de control interno. En los últimos años se han elaborado y difundido en varios países una serie de informes que presentan un enfoque integrador sobre el control interno, es decir, se lo interpreta como un sistema que abarca y atraviesa la organización en todas sus áreas, operaciones y funciones. Cabe mencionar los más conocidos:

Informe CoCo: elaborado por la junta de criterios de control del Instituto Canadiense de Contadores Matriculados (The Canadian Institute of Chartered Accountants), publicado en 1995; informe Cadbury, producido por el Cadbury Committee (Reino Unido) sobre control interno e información financiera ("Internal control and financial reporting"). El control interno permitirá que todas las empresas tengan a nivel mundial un mejor control en las diferentes áreas y logren sus objetivos trazados.

Según Pérez (2007), los componentes del nuevo enfoque del sistema de control interno (entorno de control, evaluación de los riesgos, actividades de control, información, comunicación y supervisión) no están vinculados entre sí, es decir, no generan una sinergia $y$, por tanto, no forman un sistema integrado que responda de una manera dinámica a las circunstancias cambiantes y exigentes del interno y del entorno de las entidades.

Flores \& Ibarra (2006) indica que la mayoría de las pequeñas y medianas empresas no cuentan con un control interno. Debido a que la mayoría de estas empresas son familiares, no se dispone de gente profesional que oriente cómo debe llevarse el control interno dentro de estas, pues en las empresas familiares trabajan personas que no tienen mucha idea del manejo de la empresa; además por la confianza que representa ser parte de la familia, el dueño no cree necesario tomar en cuenta un punto tan importante como el control interno, por lo que de manera intencional o no se puede caer en fraudes. Entre los aspectos más importantes que afectan a las pequeñas y medianas empresas está la falta de formalidad por la carencia de una organización adecuada a causa de que no cuenta con un manual de procedimientos y de políticas que conozcan todos los miembros de la empresa.

Henri Fayol (1841) definía el control como "la verificación de si todo ocurre en una empresa conforme al programa adoptado, a las órdenes dadas y a los principios admitidos". El control requiere la existencia de un objetivo y la aplicación de unas medidas 
correctivas. Esto ha sido la filosofía del control por objetivos, ampliamente desarrollada por Peter Drucker, y comúnmente aceptada en la actualidad por las empresas.

El control es considerado en la filosofía empresarial moderna como el análisis permanente de las desviaciones entre objetivos y realizaciones, y la adopción de las medidas correctoras que permitan cumplir con los objetivos o bien su adaptación necesaria

Gauthier (2014). se refiere al control interno, en todas sus formas, ya que desempeña un papel fundamental en las finanzas de las administraciones locales. Los auditores internos contribuyen a asegurar la integridad de la estructura de controles internos de la administración y asisten a la gestión administrativa en el cumplimiento de sus responsabilidades. Los auditores financieros externos brindan a los usuarios de los estados financieros de la administración local la seguridad independiente que necesitan: que se puede confiar en los datos en esos estados financieros. Los auditores del desempeño, tanto externos como internos, contribuyen a asegurar que los programas, las actividades y las funciones se manejen de manera eficiente y eficaz. La auditoría permite que las administraciones locales marquen el contraste entre lo que es y lo que debería ser al hacerlo. Las administraciones locales deberían estas mejor preparadas para responder a las necesidades de sus ciudadanos y a otros con un interés vital en las finanzas de la administración.

Por lo que es necesario caracterizar el control interno en la gestión de las empresas comerciales del Perú. Por lo anteriormente expresado, el enunciado del problema de investigación es el siguiente: ¿cuáles son las características del control interno en la gestión de las empresas en el Perú, caso empresas comerciales periodo 2013? Para responder a la pregunta se ha planteado el siguiente objetivo general: Identificar y describir la caracterización del control interno en la gestión de las empresas comerciales del Perú en el periodo 2013. Para poder conseguir el objetivo general nos hemos planteado los siguientes objetivos específicos: identificar y describir las características del control interno en las empresas comerciales del Perú en el periodo 2013, e identificar y describir el control interno en la gestión de las empresas comerciales periodo 2013. Finalmente, se justifica la presente investigación porque da a conocer cómo se caracteriza el control interno en la gestión de las empresas comerciales del periodo 2013. Si bien es cierto a través del control interno se mejorarán los procesos contables, administrativos y económicos que sustentarán una información veraz y precisa, este estudio permitirá un mejor entendimiento del control interno adoptando medidas dentro de la empresa, con el fin de verificar la confiabilidad de los datos contables, y porque con una mejor organización se podrá brindar un servicio de calidad.

Finalmente los resultados de esta investigación servirán de antecedente para posteriores trabajos de investigación de naturaliza similar o afín. 


\section{METODOLOGÍA}

\section{Tipo y nivel de la investigación de la tesis}

Teniendo en cuenta la complejidad para recolectar información de carácter económico y tributario en las entidades, debido a que algunas empresas guardan en absoluta reserva la información de sus actividades, el método de investigación que se aplicó es la revisión bibliográfica y documental.

El tipo de investigación es bibliográfica porque solo se limitó a investigar datos de las fuentes de información originales, sin entrar en elcuanto a detalle de cantidades.

El nivel de estudio es descriptivo porque se realizó una investigación de características, cualidades y atributos del tema de investigación sin entrar a los grados de análisis cuantitativos del problema.

\section{Diseño de la investigación}

Es muy importante utilizar un diseño de investigación teniendo en cuenta la técnica de revisión documental y bibliográfica. En la investigación es necesario definirlo a través del aporte de algunos investigadores:

La investigación realizó la búsqueda de información proveniente de propuestas, comentarios, análisis de temas parecidos al nuestro, los cuales se analizaron y ayudaron a la determinar los resultados.

El diseño de investigación aplicado es no experimental

\section{El universo o población}

Por ser una investigación con diseño de revisión documentaria, no es necesario considerar la población ni la muestra.

\section{Plan de análisis}

De acuerdo con la naturaleza de la investigación, el análisis de los resultados se hizo teniendo en cuenta la comparación de los comentarios o estudios realizados en las informaciones recolectadas.

\section{Técnicas e instrumentos de recolección de información}

La técnica que se aplicó para buscar información de los documentos de origen es la de revisión bibliográfica y documental, que para algunos investigadores tiene las siguientes definiciones: 
En cuanto a los instrumentos utilizados, teniendo en cuenta las fuentes de información documentaria y bibliográfica, como textos, tesis, páginas de internet, se elaboraron fichas bibliográficas como instrumento de ubicación de la información.

Al aplicar la técnica de la recolección de información se recurrió a las fuentes de información de origen para obtener datos, los cuales permitieron formular resultados, análisis de resultados y las conclusiones.

\section{RESULTADOS}

\section{Respecto a identificar y describir la caracterización del control interno en la gestión de las empresas comerciales del Perú en el periodo 2013.}

$>$ El control interno permite evaluar el grado de eficiencia, eficacia, economía y productividad en las empresas del rubro comercial, y lograr en algunos casos alcanzar el $100 \%$ de sus objetivos y metas programados. También permite minimizar riesgos y errores o irregularidades en un $80 \%$ de forma oportuna contando con un adecuado y eficiente control para tomar decisiones en la vida empresarial. Los estudios señalados por diversos investigadores, como Salazar (2012) y Chiavenato I. (1997), señalan que una empresa comercial (y de todo sector-actividad) que contemple un adecuado control interno dentro de sus funciones reduce en $15 \%$ los procedimientos innecesarios y aumenta la productividad de la empresa en $25 \%$.

> El sector comercial creció en el 2013 en 5,5 \% (fuente: larepublica.pe 2014), lo que ayudó a la economía nacional, pues llegó a determinar que toda empresa que se dedica a la compraventa de bienes debe tener un buen manejo y control interno de sus inventarios y procedimientos, quepues eso permitirá preservar los bienes contra pérdidas por siniestro y deterioro, ya que estos representan inversiones dentro del activo corriente. Por ello se deben tomar medidas correctivas que nos permitan traer beneficios brindando servicio de calidad. En ese sentido, efectuar el control interno nos permite corregir esos errores en casi un $80 \%$ en algunos casos.

$>$ El control interno constituye una herramienta de apoyo para que tomare decisiones la alta dirección, tanto en instituciones públicas como privadas, cuyo propósito es que los objetivos planteados por la empresa se cumplan a cabalidad con las normas y los reglamentos establecidos. Según el análisis de esta investigación científica, al aplicar un procedimiento de control interno se analiza si los objetivos de las empresas se cumplen. Al aplicar el control interno, muchos logran el $100 \%$ de sus metas y objetivos trazados; , no obstante el porcentaje de resultados varía según el giro de la entidad y el tipo de control aplicado.

$>$ El control interno comprueba la eficacia administrativa y financiera en el coordinado manejo de los recursos para lograr los objetivos y las metas previamente 
determinados. Es la evaluación entre la misión, la visión, los objetivos, las metas, los planes y las políticas establecidos, y los logros obtenidos o dejados de realizar que se configuran en beneficios económicos, sociales y de procedimientos. Según estudios, la eficiencia de los controles internos se puede apreciar en un 80 \% de su aplicación. Por otro lado, el 77 \% (información recolectada № 10, Salazar -2004-) de las empresas comerciales pequeñas del país no realiza un control interno, y deja este proceso solo en manos de empresas de gran envergadura, lo cual muestra la diferencia en la calidad de productos y procedimientos respecto a las empresas que sí realizan control interno.

\section{Respecto a identificar y describir el control interno en la gestión de las empresas comerciales en el periodo 2013.}

$>$ La deficiencia en el control interno en las diversas áreas de las empresas afecta su rentabilidad en al menos $20 \%$, según muchos analistas (entre ellos Ediciones Caballero Bustamante 2013). Por ello se debe diseñar un manual de procedimientos y funciones de su personal por cada área. El personal del área de compras debe ser capacitado para que cumpla con el desempeño de sus funciones, lo que permitirá a la empresa reducir sus gastos. Determinar un porcentaje real de cuánto afecta no aplicar el control interno en las empresas representaría una cifra errada, puesto que el análisis de causa-efecto se debe dar en específico para cada entidad, y no a nivel sector.

> Por otro lado, las empresas de carácter comercial buscan aplicar el control interno para subsanar errores, pero no constante ni cotidianamente. Por ello muchas veces el control interno o la aplicación de soluciones son solo momentáneas, y no periódicas. En ese sentido, los especialistas señalan que para que un control interno tenga un efecto en la gestión debe realizarse por lo menos semestralmente, y debe cumplir con solucionar en un $15 \%$ los errores de cada entidad. El porcentaje cambiará dependiendo de la cantidad de procedimientos de control que ejecutemos.

$>$ El control que se realiza sea de cualquier tamaño nos llevará al éxito. Por ello la empresa implementará un sistema de control interno que le ofrezca rentabilidad y mejoramiento, toda vez que las políticas de control interno permitirán que se cumplan con las funciones para cada miembro de la empresa y los objetivos que esta se ha trazado para llegar al éxito.

\section{DISCUSIÓN}

Respecto a identificar y describir la caracterización del control interno en la gestión de las empresas comerciales del Perú en el periodo 2013

El resultado obtenido coincide con lo expuesto por lo publicado en el diario Señal Alternativa (2012) y con mi caracterización (Romero 2005). Ambos coinciden con que 
cerca de un $77 \%$ de las pequeñas empresas no realiza procedimientos de control interno. Y este proceso se deja para las empresas de mayor envergadura, las que muestran una eficiencia en los procedimientos de control interno en un $80 \%$

De acuerdo con Domingo (2007) y Bilick (2003), un buen sistema de control interno permite a cualquier organización tener mayores posibilidades de lograr los objetivos que ella misma se fija, y alcanzar hasta un $100 \%$ de lo trazado, con lo que se logra la eficacia de su sistema de control interno.

Este resultado complementa lo concluido por Sánchez (2013): el sector comercio creció un 5,5\% en el 2013 al crecimiento de la economía llegando a determinar que toda empresa que se dedica a la compraventa de bienes es importante que tenga un buen manejo y control de sus inventarios, lo cual le permitirá preservar los activos contra pérdidas por siniestro y deterioro, ya que estos representan inversiones dentro del activo corriente.

\section{Respecto a la caracterización del control interno en la gestión de las empresas comerciales}

Para terminar con este análisis, Ramírez y Ramírez (2012) concluyen que el éxito de cualquier empresa se circunscribe a evaluar las actividades y el desempeño a fin de cumplir con los objetivos propuestos. Las políticas de control interno servirán de base para elaborar manuales de funciones, políticas y procedimientos a fin de mejorar el control interno en la empresa, mitigar los riesgos y cumplir con los objetivos estratégicos, operativos, de información y de cumplimiento propuestos.

Este resultado complementa lo que dicen Vargas (2004) y Méndez (2005): que el control interno es una herramienta que facilita datos sobre la razonabilidad de la información financiera y económica de las empresas cooperativas de servicios múltiples, lo que facilitará la planeación, la toma de decisiones y el control empresarial.

Y finalmente para Mellano (2005) no es suficiente cumplir con el proceso de control interno, sino que es necesario evaluar el trabajo de los auditores para asegurar un desarrollo eficiente y efectivo. El control de calidad aplicado a la auditoría es la garantía de un trabajo con los mejores estándares.

\section{CONCLUSIONES}

Luego de la revisión bibliográfica y su análisis se llegó a las siguientes conclusiones:

- La implementación de un sistema de control influye en las diferentes áreas de la empresa maximizando oportunidad, eficiencia de las operaciones, rentabilidad y confiabilidad de la información administrativa, contable y financiera, en muchos casos en un $100 \%$. El entorno o el ambiente forma al personal que desarrolla sus actividades 
y cumpla con sus responsabilidades. Las actividades de control se establecen para ayudar a asegurar que se pongan en práctica las reglas para enfrentar cualquier riesgo. Todo este proceso se supervisa para proporcionar un grado de seguridad razonable en los objetivos de la empresa.

- Con un buen sistema de control interno de inventario se reportarán en el momento oportuno los activos circulantes, de tal manera que ncubran las necesidades de sus clientes. Se debe seleccionar al personal que esté capacitado para cumplir con sus funciones, lo que permitirá reportar oportunamente las operaciones para una mejor gestión en el control y manejo de los recursos de la empresa.

- Toda empresa debe realizar procedimientos de control interno que favorezcan a tomar decisiones independientes del rubro al cual esta pertenezca. De otro lado, el control interno se caracteriza por ayudar a la gestión a corregir todos los errores que muestre la empresa en los diversos procedimientos internos. En este sentido, las empresas que apliquen el control interno podrían experimentar incremento en la productividad en cerca del $80 \%$.

- En toda organización grande o pequeña se deben implementar procesos de control interno. Para mejorar la gestión de las operaciones es necesario efectuar un control que permita procesar la información de manera exacta y oportuna a la casa matriz de la empresa y a terceros (entidad bancaria y proveedores). Dentro de una empresa es importante analizar los datos que se relacionan con las operaciones, entre los que se pueden mencionar el control de caja y bancos, cuentas por cobrar, cuentas por pagar, que cuente con un adecuado control interno para lograr los objetivos de la empresa.

- Realizar procedimientos de control interno en todas las áreas de la organización permite alcanzar sus objetivos casi en un $100 \%$, sin embargo se requiere el apoyo indispensable de la autoridad máxima de la organización para llevarse a cabo; todas sus actividades se interrelacionan de manera sistemática y brindan información confiable a todos sus grupos de interés.

- Con la deficiencia en el control interno del área de compras y del área contable que afecta el área de ventas y la rentabilidad de la empresa, se debe diseñar un manual de procedimientos y funciones del personal por cada área. El personal debe ser capacitado para que cumpla con el $100 \%$ de desempeño de sus funciones, y debe haber una buena organización administrativa: cada compra debe estar con su cotización y orden de compra.

- En aquellas empresas que no realicen procesos de control interno se debe reestructurar esa política y hacerlo de manera que se apliquen estos procedimientos y se pueda mejorar la gestión de la entidad. Cuando una empresa no efectúa controles internos ve mellada su productividad en un $25 \%$. 


\section{REFERENCIAS BIBLIOGRÁFICAS}

Balarezo J. (2013). El servicio de transporte interprovincial de pasajeros en Áncash (E. Saavedra, entrevistador).

Bilick G. (2013). Boletín de la Comisión de Normas y Asuntos Profesionales del Instituto de Auditores Internos de Argentina. [recuperado el 30 de enero del 2013]. Disponible en: https://www.iaia.org.ar/revistas/normaria/Normaria05.pdf

Domingo C. (2007). La efectividad del control interno en el buen gobierno de las empresas de transporte urbano. Recuperado de http://www.bibliomaster.com/pdf/731.pdf

Estrada W. (2013). Ventajas en la implementación de un sistema de control interno en el área de almacén de la Ugel, Sihuas. Chimbote: Universidad Católica Los Ángeles de Chimbote.

Flores Y. e Ibarra G. (2006). Diseño de control interno para la pequeña y mediana empresa. Pachuga: Universidad Autónoma del estado de Hidalgo, Instituto de Ciencias Económico-Administrativas.

Ramírez A. y Ramírez J. (2012). Planificación estratégica y propuesta de políticas de control interno para la empresa general Logistic and Services S.A. basadas en el objetivo estratégico y operativo de administración de riesgo empresarial. Guayaquil, Ecuador: Universidad Politécnica Salesiana.

Sánchez E. (2013). La optimización del sistema de control interno de inventarios en el departamento de almacén de las empresas comerciales del Perú., Chimbote, Del Santa, Perú: Universidad Católica Los Ángeles de Chimbote.

García M. (2005). El nuevo rol del auditor financiero en las empresas de servicios. Buenos Aires.

Guardia. (2006). La auditoría de gestión como herramienta del control y evaluación del gobierno local de Angaraes, Lircay.

Gómez. (2000). El control de gestión como herramienta fundamental para la misión financiera. Lima.

Julián F. (2007). La contabilidad y la auditoría financiera.

Oviedo P. (2010). Auditoría financiera. [consultada el 8 de julio del 2013]. Disponible en: http://www.monografias.com/trabajos60/auditoria-financiera/auditoria-financiera.shtml. 